\title{
LIE LOOPS WITH INVARIANT UNIFORMITIES. II
}

\author{
BY \\ SIGMUND N. HUDSON
}

This paper presents another solution to the so-called Hilbert's Fifth Problem, the problem of determining on which locally euclidean algebraic structures can an analytic structure be introduced so that the algebraic operations are analytic mappings. Algebraic structures for which such analytic structures exist are called Lie. Below we give a generalization to the theorem that a locally euclidean group is a Lie group (see [2] and [5]). Our generalization moves in the direction of nonassociativity. We prove the theorem that a diassociative locally euclidean loop with a right invariant uniformity and a left invariant uniformity is a Lie loop.

The background material for this paper can be found in [4]. $\$ 2$ of [4] will be particularly helpful concerning definitions, notations, and introductory propositions. As in [4], the techniques are those of analytic transitive transformation groups and Lie group theory; and the proof of our theorem depends upon the theorem being generalized.

In [4] it is proved that a locally euclidean loop with an invariant uniformity is a Lie loop. Thus we have weakened the assumptions relating to uniform structures, but have, in turn, added the diassociativity condition. It still seems plausible that the assumption of diassociativity might be dropped without loss of generality in our theorem, in spite of its heavy use in the proof below.

In the proof of the following lemmas and the theorem $\mathscr{G}$ - denotes the group of right translates of a loop $\mathscr{L}, \mathscr{H}$ - the group of left translates of $\mathscr{L}, \mathscr{G}$ the group generated by the right translates, and $\mathscr{H}$ the group generated by the left translates [4, Definitions 2.1 and 2.3]. The groups $\mathscr{G}-$ and $\mathscr{H}$ - are Lie groups of homeomorphisms acting effectively and transitively on $\mathscr{L}$ when $\mathscr{L}$ is locally euclidean with a right invariant uniformity and a left invariant uniformity [4, Proposition 2.5]. We let $R_{x}$ and $L_{x}$ denote the right and left translates, respectively, by $x \in \mathscr{L}\left(R_{x}(y)=y x\right.$, for example). The Lie algebra of $\mathscr{G}$ - is denoted by $\mathfrak{L}\left(\mathscr{G}^{-}\right)$; we assume that the usual identification exists between it and the tangent space $T_{e}$ to $\mathscr{G}^{-}$at $e$ (the identity of $\mathscr{G}^{-}$)[3, p. 89]. The well-known exponential mapping from $\mathscr{L}\left(\mathscr{G}^{-}\right)$into $\mathscr{G}^{-}$is denoted by Exp. The homeomorphisms $C_{R}$ and $C_{L}$ are the mappings of $\mathscr{L}$ into $\mathscr{G}-$ and $\mathscr{H}-$, respectively, defined by $C_{R}(x)=R_{x}$ and $C_{L}(x)=L_{x}$, respectively. The solution for $x$ in the equation $z x=b$ in a loop is usually denoted by $z^{(-1)} b$. However, in a diassociative loop (a loop in which every two elements lie in a subgroup) it is possible to define, in an obvious manner, for each element $z$ an element $z^{-1}$ so that

Presented to the Society, August 27, 1964; received by the editors July 13, 1964. 
$z^{(-1)} b=z^{-1} \cdot b$, i.e., the parentheses enclosing -1 can be dropped. It is also true that $\left(R_{x}\right)^{-1}=R_{x^{-1}}$.

The following lemmas break up the length of the proof of the theorem.

Lemma 1. Let $\mathscr{L}$ be a diassociative loop. If $\left(\cdots\left(a_{1} a_{2}\right) \cdots\right) a_{n}=1,1$ being the identity of $\mathscr{L}$, then $a_{1}\left(\cdots\left(a_{n-1} a_{n}\right) \cdots\right)=1$ for $a_{i} \in \mathscr{L}$.

Proof. For $n=3$, if $\left(a_{1} a_{2}\right) a_{3}=1$, then $a_{3}$ belongs to the group generated by $a_{1}$ and $a_{2}$. Hence $1=\left(a_{1} a_{2}\right) a_{3}=a_{1}\left(a_{2} a_{3}\right)$, and the lemma is true. Assume that the lemma is true for all products of $n$ or less elements of $\mathscr{L}, n \geqq 3$. If $\left(\cdots\left(a_{1} a_{2}\right) \cdots\right) a_{n+1}=1$, then $\left(\cdots\left(a_{1} a_{2}\right) \cdots\right) a_{n-1}=a_{n+1}^{-1} a_{n}^{-1}$. There exists $x \in \mathscr{L}$ such that $\left(\left(\cdots\left(a_{1} a_{2}\right) \cdots\right) a_{n-1}\right) x=\left(a_{n+1}^{-1} a_{n}^{-1}\right) x=1$. By the induction hypothesis, $a_{1}\left(a_{2}\left(\cdots\left(a_{n-1} x\right) \cdots\right)\right)=1$. Also $x=a_{n} a_{n+1}$. Hence $a_{1}\left(a_{2}\left(\cdots\left(a_{n} a_{n+1}\right) \cdots\right)\right)$ equals 1 .

COROLlaRY 1. If $\mathscr{L}$ is diassociative and if $R_{a_{n}} \cdots R_{a_{1}}(1)=1$, then $L_{a_{1}} \cdots L_{a_{n}}(1)=1$. (Recall that $R_{a_{1}} \cdots R_{a_{n}}$ is the composite mapping that maps $x$ upon $\left(\left(\cdots\left(x a_{1}\right) \cdots\right) a_{n}\right)$

LEMMA 2. Let $\mathscr{L}$ be a locally compact, connected, diassociative loop with a right invariant uniformity and a left invariant uniformity. Then there is a bicontinuous anti-isomorphism $\phi$ from the group of right translates $\mathscr{G}^{-}$ onto the group $\mathscr{H}^{-}$of left translates so that $\phi\left(R_{x}\right)=L_{x}$ and $\phi\left(\mathscr{G}_{1}^{-}\right)=\mathscr{H}_{1}^{-}$.

Proof. The groups $\mathscr{G}^{-}$and $\mathscr{H}^{-}$are (locally compact) transformation groups with the $g$-topology acting effectively and transitively on $\mathscr{L}$ [4, Proposition 2.2]. For $x \in \mathscr{L}$, define $\phi\left(R_{x}\right)=L_{x}$; then extend the domain of $\phi$ to $\mathscr{G}$, the group generated by the right translates, by the requirement that $\phi$ be an algebraic antiisomorphism. That is, if $R_{x_{1}} \cdots R_{x_{n}} \in \mathscr{G}$, then $\phi\left(R_{x_{1}} \cdots R_{x_{n}}\right)=L_{x_{n}} \cdots L_{x_{1}}$. It must be shown that $\phi$ is a well-defined function. Suppose $R_{x_{1}} \cdots R_{x_{n}}$ and $R_{x_{n+1}} \cdots R_{x_{m}}$ are the same homeomorphisms of $\mathscr{L}$. Then $R_{x_{m}}^{-1} \cdots R_{x_{n+1}}^{-1} R_{x_{1}} \cdots R_{x_{n}}\left(x^{-1}\right)=x^{-1}$ for all $x \in \mathscr{L}$, implying that $R_{x} R_{x_{m}}^{-1} \cdots R_{x+1}^{-1} R_{x_{1}} \cdots R_{x_{n}} R_{x}^{-1}(1)=1$. By Corollary 1 , $L_{x}^{-1} L_{x_{n}} \cdots L_{x_{1}} L_{x_{n}+1}^{-1} \cdots L_{x_{m}}^{-1} L_{x}(1)=1$ for all $x$, making $L_{x_{n}} \cdots L_{x_{1}} L_{x_{n+1}}^{-1} \cdots L_{x_{m}}^{-1}(x)=x$ for all $x$, and $L_{x_{n}} \cdots L_{x_{1}}(x)=L_{x_{m}} \cdots L_{x_{n+1}}(x)$ for all $x$. Thus $\phi$ is well defined. Clearly, $\phi$ is onto $\mathscr{H}$. To show that $\phi$ is one-to-one on $\mathscr{G}$, it is sufficient to show that if $R_{a_{1}} \cdots R_{a_{n}}(x) \neq x$ for some $x \in \mathscr{L}$, then $L_{a_{n}} \cdots L_{a_{1}}(y) \neq y$ for some $y \in \mathscr{L}$. Assuming $R_{a_{1}} \cdots R_{a_{n}}(x) \neq x$, we have that $R_{a_{1}} \cdots R_{a_{n}} R_{x}(1) \neq x$ and that $R_{z} R_{a_{1}} \cdots R_{a_{n}} R_{x}(1)=1$ for some $z$ in $\mathscr{L}$, with $z \neq x^{-1}$. Then, by Corollary 1 , $L_{x} L_{a_{n}} \cdots L_{a_{1}} L_{z}(1)=1$ and, finally, $L_{a_{n}} \cdots L_{a_{1}}(z)=x^{-1} \neq z$.

It will next be shown that if $\left\{f_{\delta}\right\}$ is a net in $\mathscr{G}$ and if $f_{\delta} \rightarrow f \in \mathscr{G}^{-}$, then $\phi\left(f_{\delta}\right)$ converges; furthermore, if $f$ also belongs to $\mathscr{G}$, then $\phi\left(f_{\delta}\right) \rightarrow \phi(f)$. Let $\left\{x_{\beta}\right\}$ be a net in $\mathscr{L}$ converging to $x$. For each $\beta$ and $\delta$ there exists an element $t_{\delta \beta} \in \mathscr{L}$ such that $t_{\delta \beta}\left[\phi\left(f_{\delta}\right)\right]\left(x_{\beta}\right)=1$. By Lemma $1, t_{\delta \beta}\left[\phi\left(f_{\delta}\right)\right]\left(x_{\beta}\right)=f_{\delta}\left(t_{\delta \beta}\right) x_{\beta}=1$. Hence $\left(t_{\delta \beta}\left[\phi\left(f_{\delta}\right)\right]\left(x_{\beta}\right)\right) x_{\beta}^{-1}=f_{\delta}\left(t_{\delta \beta}\right)=x_{\beta}^{-1}$ and $t_{\delta \beta}=f_{\delta}^{-1}\left(x_{\beta}^{-1}\right)$. Since $x_{\beta} \rightarrow x$ and $f_{\delta} \rightarrow f$, it follows that $x_{\beta}^{-1} \rightarrow x^{-1}, f_{\delta}^{-1} \rightarrow f^{-1}$, and $t_{\delta \beta} \rightarrow f^{-1}\left(x^{-1}\right)$. Hence $\phi\left(f_{\delta}\right)$ converges (in the $g$-topology). If $f$ also belongs to $\mathscr{G}$, then $f_{\delta}\left(t_{\delta \beta}\right) x_{\beta}=1$ implies that 
$f\left(f^{-1}\left(x^{-1}\right)\right) x=1$ and that $(*) f^{-1}\left(x^{-1}\right)[\phi(f)](x)=1$. On the other hand, letting $\lim _{\delta} \phi\left(f_{\delta}\right)=h$, it follows that $(* *) 1=\lim _{\delta, \beta} t_{\delta \beta}\left[\phi\left(f_{\delta}\right)\right]\left(x_{\beta}\right)=f^{-1}\left(x^{-1}\right) h(x)$. From $(*)$ and $(* *)$ it follows that $[\phi(f)](x)=h(x)$. Summarizing our conclusions, $\phi$ is a continuous anti-isomorphism from $\mathscr{G}$ onto $\mathscr{H}$. Also, $\phi$ is uniformly continuous. Consequently, the domain of $\phi$ may be further extended to $\mathscr{G}^{-}$such that $\phi$ is a continuous anti-homomorphism from $\mathscr{G}$ - into $\mathscr{H}-$. It can be easily shown that $\phi$ is one-to-one (by a limiting argument and with the use of Lemma 1 ), and an argument dual to that of the continuity of $\phi$ shows that $\phi^{-1}$ is continuous, as well as one-to-one. Thus $\phi$ is a bicontinuous anti-isomorphism from $\mathscr{G}^{-}$onto $\mathscr{H}$ -

From Lemma 1 and its corollary it follows that $\phi\left(\mathscr{G}_{1}\right)=\mathscr{H}_{1}$; this implies that $\phi\left(\mathscr{G}_{1}^{-}\right)=\mathscr{H}_{1}^{-}$.

LemMA 3. Let $\mathscr{G}, \mathscr{G}-, \mathscr{H}-, \mathscr{L}$, and $\phi$ be as in Lemma 2. Let I: $\mathscr{H}-\rightarrow \mathscr{H}^{-}$ map $h$ onto $h^{-1} \in \mathscr{H}^{-}$. If $g \in \mathscr{G}^{-}$and $g(1)=y$, then $[I(\phi(g))](1)=y^{-1}$.

Proof. The lemma is first proved for $g \in \mathscr{G}$. If $g=R_{x}$ for some $x \in \mathscr{L}$, then the lemma is true. Suppose the lemma is true for all products of $n$ or less right translates, $n \geqq 1$. If $g(1)=y$ and if $g=R_{x_{n+1}} \cdots R_{x_{1}}$, then $I(\phi(g))=L_{x_{n+1}}^{-1} \cdots L_{x_{1}}^{-1}$. Let $h$ denote $R_{x_{n}} \cdots R_{x_{1}}$. Then $h(1)=g(1) x_{n+1}^{-1}$. By the induction hypothesis, $[I(\phi(h))](1)=x_{n+1} g(1)^{-1}$. However, $[I(\phi(g))](1)=\left[L_{x_{n+1}}^{-1} I(\phi(h))\right](1)$. Therefore, $[I(\phi(g))](1)=x_{n+1}^{-1}\left(x_{n+1} g(1)^{-1}\right)=g(1)^{-1}$, as desired. The lemma is true for $g \in \mathscr{G}$ - using a limit argument.

LEMMA 4. Let $\mathscr{L}$ be a connected, diassociative, locally euclidean loop with a right invariant uniformity. Let $\mathscr{M}$ be the natural quotient analytic structure obtained from $\mathscr{G}$ - on both $\mathscr{G}_{-} / \mathscr{G}_{1}^{-}$and $\mathscr{L}$ (since $\mathscr{L}$ is homeomorphic with $\left.\mathscr{G}^{-} \mid \mathscr{G}_{1}^{-}\right)$. Then there is an "exponential" mapping exp from the tangent space $T_{1}$ at the identity 1 of $\mathscr{L}$ into $\mathscr{L}$ so that $\exp (s+t) X=\exp s X \exp t X$ and $\exp -X=(\exp X)^{-1}$ for $X \in T_{1}$ and real numbers $s$ and $t$. The mapping exp is analytic with respect to $T_{1}$ and $\mathscr{M}$, and $\exp$ is a local analytic isomorphism at the origin of $T_{1}$.

Proof. We denote the algebra of real-valued locally analytic functions on $\mathscr{L}$ by $\mathfrak{U}(\mathscr{L})$. We will first show that, for each $X \in T_{1}$, there is a unique (analytic) oneparameter subgroup $\pi$ of $\mathscr{L}$ so that $X(f)=d f(\pi(t)) /\left.d t\right|_{(t=0)}$ for $f \in \mathfrak{A}(\mathscr{L})$, i.e., $\pi$ has $X$ as a tangent vector at $1 \in \mathscr{L}$. Unique means here that $\pi_{1}(t)=\pi_{2}(t)$ for all $t$ in some neighborhood of zero implies that $\pi_{1}=\pi_{2}$. One easily verifies that, if $\pi(t)$ has $X$ as tangent vector at $1 \in \mathscr{L}$, then $\pi(a t)$ has $a X$ as tangent vector at 1 for any real number $a$. Let $\mathscr{V}=\left\{X \in T_{1}: X\right.$ is the tangent vector at 1 for some local one-parameter subgroup of $\mathscr{L}\}$. It suffices to show that $\mathscr{V}$ contains an $n$-dimensional subset (containing the zero vector) of $T_{1}, n$ being the dimension of $\mathscr{L}$; for if $X \in \mathscr{V}$, then $a X$ clearly belongs to $\mathscr{V}$ for any real number $a$. Let $A$ be some neighborhood of the zero vector of $\mathscr{L}\left(\mathscr{G}^{-}\right)$on which Exp is one- 
to-one and $U$ be a neighborhood of $1 \in \mathscr{L}$ so that $C_{R}(U) \subset \operatorname{Exp} A$. The mapping $\operatorname{Exp}^{-1} C_{R}$ defined on $U$ is one-to-one and continuous; hence, $\operatorname{Exp}^{-1} C_{R}(U)$ is an $n$-dimensional subset of $\mathfrak{L}\left(\mathscr{G}^{-}\right)$.

Let $X_{n+1}, \cdots, X_{r}$ be $r-n$ linearly independent vectors in $\mathscr{L}\left(\mathscr{G}^{-}\right)$so that $\operatorname{Exp} X_{i} \in \mathscr{G}_{1}^{-}$and the vectors $X_{i}$ span $\mathfrak{L}\left(\mathscr{G}_{1}^{-}\right) \subset \mathfrak{Q}\left(\mathscr{G}^{-}\right), n+1 \leqq i \leqq r$. Also, let $X_{1}, \cdots, X_{n}$ be $n$ linearly independent vectors in $\mathfrak{L}\left(\mathscr{G}^{-}\right)$so that $\operatorname{Exp} t X_{j}=R_{\pi_{j}(t)}$ for some one-parameter subgroup $\pi_{j}(t)$ in $\mathscr{L}, 1 \leqq j \leqq n$. The fact that the vectors $X_{j}, 1 \leqq j \leqq n$, may be so chosen is a consequence of the following statements: $R_{\pi(t)}$ is a one-parameter subgroup of $\mathscr{G}$ - for each one-parameter subgroup $\pi(t)$ in $\mathscr{L}, \mathscr{L}$ has a neighborhood of 1 uniquely covered by one-parameter subgroups [4, Theorem 3.6], and $C_{R}(U)$ is an $n$-dimensional subset of $\mathscr{G}$-. One easily shows that $\left\{X_{i}: 1 \leqq i \leqq r\right\}$ spans $\mathscr{L}\left(\mathscr{G}^{-}\right)$. If $P$ and $Q$ belong to $\operatorname{Exp}^{-1} C_{R}(\mathscr{L})$, then we may assume the following: $P=p_{1} X_{1}+\cdots+p_{r} X_{r}$ and $Q=q_{1} X_{1}+\cdots$ $+q_{r} X_{r}, \operatorname{Exp} P=R_{p}=R_{\pi_{1}\left(p_{1}\right)} \cdots R_{\pi_{n}\left(p_{n}\right)} h_{n+1} \cdots h_{r}, \operatorname{Exp} Q=R_{q}=R_{\pi_{1}\left(q_{1}\right)} \cdots R_{\pi_{n}\left(q_{n}\right)} h_{n+1}^{\prime} \cdots h_{r}^{\prime}$ for some $p$ and $q$ in $\mathscr{L}$, some real numbers $p_{i}$ and $q_{i}$, and some $h_{i}$ and $h_{i}^{\prime}$ in $\mathscr{G}_{1}^{-}$. If we furthermore assume that $P \neq Q$, then $R_{p} \neq R_{q}$ and $p \neq q$. Hence the vectors $\left(p_{1}, \cdots, p_{n}\right)$ and $\left(q_{1}, \cdots, q_{n}\right)$ cannot be equal (for otherwise $p=q)$. Letting $(d \psi)_{e}$ denote the differential of the mapping $\psi: \mathscr{G}-\rightarrow \mathscr{L}$ defined by $\psi(g)=g(1)$ (note that $(d \psi)_{e}$ is a mapping from $\mathscr{L}\left(\mathscr{G}^{-}\right)$onto $T_{1}$ ) we have shown that, for two distinct tangent vectors $P$ and $Q$ in $\operatorname{Exp}^{-1} C_{R}(\mathscr{L})$, it is true that $(d \psi)_{e} P \neq(d \psi)_{e} Q$. Hence $B=(d \psi)_{e} \operatorname{Exp}^{-1} C_{R}(U)$ is an $n$-dimensional subset of $T_{1}$, being the one-to-one continuous image of the $n$-dimensional set $\operatorname{Exp}^{-1} C_{R}(U)$. It is easily shown that, if $X \in \mathscr{V}$ and if the one-parameter subgroup $\pi(t)$ has $X$ as its tangent vector at 1 , then $(d \psi)_{e} \operatorname{Exp}^{-1} C_{R}(\pi(1))=X$. This means that $B$ is a subset of $\mathscr{V}$, thereby showing that, for each vector $X$ in $T_{1}$, there is at least one local one-parameter subgroup having $X$ as its tangent vector. Also we see that there is only one one-parameter subgroup of $\mathscr{L}$ for each tangent vector in $T_{1}$. For if $\pi_{1}(t)$ and $\pi_{2}(t)$ are two different such subgroups belonging to the vector $X \in T_{1}$, then $R_{\pi_{1}(t)}$ and $R_{\pi_{2}(t)}$ are two different one-parameter subgroups of $\mathscr{G}^{-}$with different tangent vectors $P$ and $Q$, respectively, in $\mathscr{L}\left(\mathscr{G}^{-}\right)$. But the above argument shows that $X=(d \psi)_{e} P \neq(d \psi)_{e} Q=X$.

Making use of the one-parameter subgroups in $\mathscr{L}$, it is now possible to define an exponential mapping from $T_{1}$ into $\mathscr{L}$. We follow Chevalley [1, pp. 116118] almost word-for-word, although the structure of $T_{1}$ is not as special as that in the Lie algebra case. For example, a Lie algebra compatible with the multiplication in $\mathscr{L}$ cannot, in general, be defined on $T_{1}$; and the elements of $T_{1}$ are not necessarily left or right invariant vector fields. For $X \in T_{1}$ we have shown that there is one and only one analytic one-parameter subgroup $\pi_{X}(t)$ of $\mathscr{L}$ having $X$ as its tangent vector. Assuming, without loss of generality, that every oneparameter subgroup in $\mathscr{L}$ is defined for the real number 1 , we define $\exp X$ to be $\pi_{X}(1)$. Thus exp is a mapping from $T_{1}$ into $\mathscr{L}$. The following properties of exp obviously hold: 


$$
\begin{aligned}
\exp \left(t_{1}+t_{2}\right) X & =\left(\exp t_{1} X\right)\left(\exp t_{2} X\right), \\
\exp -t X & =(\exp t X)^{-1}
\end{aligned}
$$

We now show that exp is locally at the zero vector an analytic mapping of $T_{1}$ into $\mathscr{L}$. For $f \in \mathfrak{U}(\mathscr{L}), p \in \mathscr{L}, X \in T_{1}$, and a one-parameter subgroup $\pi$ having $X$ as its tangent vector, define $[X(f)](p)=\lim _{t \rightarrow 0}\left(\left(f\left(R_{\pi(t)}(p)\right)-f(p)\right) / t\right)$. One easily shows that $X f \in \mathfrak{U}(\mathscr{L})$ and that $X$ is a derivation of $\mathfrak{U}(\mathscr{L})$. (Also $X_{1}$, the tangent vector determined by $X$ at $1 \in \mathscr{L}$, has $\pi$ as its associated oneparameter subgroup.) We choose a coordinate system $\left(y_{1}, \cdots, y_{n}\right), y_{i} \in \mathfrak{U}(\mathscr{L})$, in a neighborhood $W$ of 1 so that $y_{i}(1)=0$ for all $i$ and so that there is a positive real number $b$ with $W=\left\{w \in \mathscr{L}:\left|y_{i}(w)\right| \leqq b\right\}$. We also choose a basis $\left\{X_{1}, \cdots, X_{n}\right\}$ of linearly independent vectors in $T_{1}$. For any real numbers $u_{1}, \cdots, u_{n}$ there is a number $\varepsilon>0$ such that $\exp t \sum u_{i} X_{i} \in W$ for $|t|<\varepsilon$. Let $T\left(u_{1}, \cdots, u_{n}\right)$ be the least upper bound of the numbers $\varepsilon$ satisfying this condition. Then define the functions $F_{j}, 1 \leqq j \leqq n$, by the rule $F_{j}\left(t, u_{1}, \cdots, u_{n}\right)=y_{j}\left(\exp t \sum u_{i} X_{i}\right)$ for $|t|<T\left(u_{1}, \cdots, u_{n}\right)$ and all $u_{1}, \cdots, u_{n}$. For each $i$ and $j, X_{i} y_{j}$ belongs to $\mathfrak{A}(\mathscr{L})$, as mentioned above. Consequently, there exist functions $U_{i j}\left(y_{1}(w), \cdots, y_{n}(w)\right)$ which are analytic and defined whenever $\left|y_{j}(w)\right| \leqq b(1 \leqq j \leqq n)$ with the property that $\left[X_{i}\left(y_{j}\right)\right](w)=U_{i j}\left(y_{1}(w), \cdots, y_{n}(w)\right)$. It follows from the definitions of $\exp$ and $X_{i} y_{j}$ that

$$
\left.\frac{d F_{j}}{d t}\right|_{\left(t, u_{1}, \ldots, u_{n}\right)}=\sum u_{i} U_{i j}\left(F_{1}\left(t, u_{1}, \cdots, u_{n}\right), \cdots, F_{n}\left(t, u_{1}, \cdots, u_{n}\right)\right),
$$

as the following argument shows:

$$
\begin{aligned}
& \left.\frac{d F_{j}}{d t}\right|_{\left(t, u_{1}, \ldots, u_{n}\right)}=\left.\frac{d y_{j}\left(\exp t \sum u_{i} X_{i}\right)}{d t}\right|_{t} \\
& =\lim _{s \rightarrow 0} \frac{y_{j}\left(\exp (s+t) \sum u_{i} X_{i}\right)-y_{j}\left(\exp t \sum u_{i} X_{i}\right)}{s} \\
& =\lim _{s \rightarrow 0} \frac{y_{j}\left(\exp t \sum u_{i} X_{i} \cdot \exp s \sum u_{i} X_{i}\right)-y_{j}\left(\exp t \sum u_{i} X_{i}\right)}{s} \\
& =\left[\left(\sum u_{i} X_{i}\right) y_{j}\right]\left(\exp t \sum u_{i} X_{i}\right)=\left[\sum u_{i}\left(X_{i} y_{j}\right)\right]\left(\exp t \sum u_{i} X_{i}\right) \\
& =\sum u_{i}\left[X_{i} y_{j}\right]\left(\exp t \sum u_{i} X_{i}\right)=\sum u_{i} U_{i j}\left(F_{1}\left(t, u_{1}, \cdots, u_{n}\right), \cdots, F_{n}\left(t, u_{1}, \cdots, u_{n}\right)\right) \text {, }
\end{aligned}
$$

all summations taken from $i=1$ to $n$. Summarizing, the functions $F_{j}, 1 \leqq j \leqq n$, satisfy the following system of differential equations with unknown functions $z_{j}$ :

$$
\frac{d z_{j}}{d t}=\sum_{i=1}^{n} u_{i} U_{i j}\left(z_{1}, \cdots, z_{n}\right) \quad(1 \leqq j \leqq n),
$$

and with boundary conditions $z_{j}\left(0, u_{1}, \cdots, u_{n}\right)=0$. Using the existence and uniqueness theory of such systems of differential equations (see [1, p. 117]), it follows that the functions $F\left(1, u_{1}, \cdots, u_{n}\right)$ are defined and analytic in some neighborhood 
of the zero vector $(0, \cdots, 0)$. Hence the mapping exp is analytic at the zero vector of $T_{1}$. Chevalley's argument $[1$, p. 118,1 st paragraph] shows that exp is locally at the zero vector an analytic isomorphism.

Lemma 5. Let $\mathscr{L}$ be a loop such that the group $\mathscr{G}$ - of right translates and the group $\mathscr{H}$ - of left translates are Lie groups. Let $\mathscr{M}$ be the natural quotient analytic structure obtained from $\mathscr{G}^{-}$on both $\mathscr{G}-\mid \mathscr{G}_{1}^{-}$and $\mathscr{L}$ (since $\mathscr{L}$ is homeomorphic with $\mathscr{G}^{-}\left(\mathscr{G}_{1}^{-}\right)$, and let $\mathscr{N}$ be the quotient analytic structure obtained from $\mathscr{H}^{-}$on both $\mathscr{H}^{-} \mid \mathscr{H}_{1}^{-}$and $\mathscr{L}$. If the identity function id from $(\mathscr{L}, \mathscr{M})$ to $(\mathscr{L}, \mathscr{N})$ is an analytic isomorphism, if $C_{R}$ mapping $(\mathscr{L}, \mathscr{M})$ into $\mathscr{G}-$ and $C_{L}$ mapping $(\mathscr{L}, \mathscr{N})$ into $\mathscr{H}$ - are both analytic, then $(\mathscr{L}, \mathscr{M})$ is a Lie loop.

Proof. With the natural analytic structures the following functions are analytic $[1$, p. 110]:

$$
\begin{aligned}
& F_{1}: \mathscr{G}^{-} \times(\mathscr{L}, \mathscr{M}) \rightarrow(\mathscr{L}, \mathscr{M}) \text { defined by } F_{1}(g, x)=g(x), \\
& F_{2}: \mathscr{H}^{-} \times(\mathscr{L}, \mathscr{N}) \rightarrow(\mathscr{L}, \mathscr{N}) \text { defined by } F_{2}(h, x)=h(x) .
\end{aligned}
$$

Let $i_{1}$ denote the identity mapping from $(\mathscr{L}, \mathscr{M})$ onto $(\mathscr{L}, \mathscr{M})$ and $i_{2}$ the identity mapping from $(\mathscr{L}, \mathscr{N})$ onto $(\mathscr{L}, \mathscr{N})$. The mapping defined on a group that maps $g$ onto $g^{-1}$ is denoted by $I$. The following composite functions from $(\mathscr{L}, \mathscr{M})$ $\times(\mathscr{L}, \mathscr{M})$ onto $(\mathscr{L}, \mathscr{M})$ conclude the proof:

$$
\begin{aligned}
& (x, y) \stackrel{\mathrm{id} \times \mathrm{id}}{\longrightarrow}(x, y) \stackrel{C_{L} \times i_{2}}{\longrightarrow}\left(L_{x}, y\right) \stackrel{F_{2}}{\longrightarrow} x y \stackrel{\mathrm{id}^{-1}}{\longrightarrow} x y \\
& (x, y) \stackrel{\mathrm{id} \times \mathrm{id}}{\longrightarrow}(x, y) \stackrel{C_{L} \times i_{2}}{\longrightarrow}\left(L_{x}, y\right) \stackrel{I \times i_{2}}{\longrightarrow}\left(L_{x}^{-1}, y\right) \stackrel{F_{2}}{\longrightarrow} x^{(-1)} y \stackrel{\mathrm{id}^{-1}}{\longrightarrow} x^{(-1)} y \\
& (x, y) \stackrel{C_{R} \times i_{1}}{\longrightarrow}\left(R_{x}, y\right) \stackrel{I \times i_{1}}{\longrightarrow}\left(R_{x}^{-1}, y\right) \stackrel{F_{1}}{\longrightarrow} y x^{(-1)} .
\end{aligned}
$$

THEOREM. Let $\mathscr{L}$ be a diassociative loop with a right invariant uniformity and a left invariant uniformity. If $\mathscr{L}$ is locally compact, locally connected, connected, and finite dimensional, then $\mathscr{L}$ is a Lie loop.

Proof. The proof consists of the verification that the hypotheses of Lemma 5 hold.

The groups $\mathscr{G}$ - of right translates of $\mathscr{L}$ and $\mathscr{H}$ - of left translates of $\mathscr{L}$ are Lie transformation groups acting effectively, transitively, and analytically on $(\mathscr{L}, \mathscr{M})$ and $(\mathscr{L}, \mathscr{N})$, respectively ([4, Proposition 2.5$]$ and Lemma 5 above).

It is first shown that the identity function id: $(\mathscr{L}, \mathscr{M}) \rightarrow(\mathscr{L}, \mathscr{N})$ is an analytic isomorphism. To this end, it will be shown in this paragraph that the mapping $M:(\mathscr{L}, \mathscr{M}) \rightarrow(\mathscr{L}, \mathscr{N})$ defined by $M(y)=y^{-1}$ is an analytic isomorphism. There exists an open set $U$ containing $1 \in \mathscr{L}$ and an analytic mapping $\beta: U \rightarrow \mathscr{G}^{-}$such that $F_{1}(\beta(x), 1)=x$ for $x \in U\left((\beta, U)\right.$ is a local cross-section with respect to $\left.\mathscr{G}_{1}^{-}\right)$ $[1$, p. 110$]$. Since $\phi$ is an analytic mapping from $\mathscr{G}-$ onto $\mathscr{H}-$, the mapping 
of $y$ onto $F_{2}(I \phi \beta(y), 1)$ defined on $U$ with the relative analytic structure is an analytic mapping into $(\mathscr{L}, \mathscr{N})$. But Lemma 3 says that $F_{2}(I \phi \beta(y), 1)=y^{-1}$. Hence the mapping $M$ is locally analytic at $1 \in \mathscr{L}$. By using right and left translates it is easily seen that $M$ is a global analytic mapping. Finally, a dual argument shows that $M^{-1}$ is an analytic mapping; hence $M$ is an analytic isomorphism.

Using the local analytic isomorphism exp of Lemma 4, it follows that there is a neighborhood $W=W^{-1}$ of 1 so that the mapping $Q:(\mathscr{L}, \mathscr{M}) \rightarrow(\mathscr{L}, \mathscr{M})$ defined by $Q(y)=y^{-1}$ is locally an analytic isomorphism at $y \in W$. Since id $=M Q$, it follows that id is a local analytic isomorphism for $y \in W$.

We next show that id is globally analytic. Since $\mathscr{G}_{1}^{-}$is compact [4, Proposition 2.4], the analytic structure $\mathscr{M}$ on $\mathscr{L}$ may be assumed to be Riemannian with Riemannian metric invariant under the action of $\mathscr{G}^{-}$, that is, elements of $\mathscr{G}^{-}$ are isometries of $\mathscr{L}$ with respect to this metric [5, p. 215]. The following techniques are due to Myers and Steenrod [6, particularly pp. 412-415]. There exist $n+1$ points $p_{0}, \cdots, p_{n}$ in some "normal" neighborhood of $1 \in \mathscr{L}$ ( $n$ is the dimension of $\mathscr{L})$ with the property that the mapping $\tau$ from $\mathscr{G}$ - into $\mathscr{L}^{n+1}\left(\mathscr{L}^{n+1}\right.$ having the product analytic Riemannian structure $\left.\mathscr{M}^{n+1}\right)$ defined by $\tau(g)=\left(g\left(p_{0}\right), \cdots, g\left(p_{n}\right)\right)$ is analytic and a homeomorphism. The points $p_{0}, \cdots, p_{n}$ may be chosen in this normal neighborhood in any manner so long as they do not all belong in the same $(n-1)$-dimensional geodesic hyperspace [6, p. 405]. Hence we may so choose them, as well as a neighborhood $V$ of 1 in $\mathscr{L}$, so that $V \cup \bigcup_{i=0}^{n} p_{i} V$ is contained in the neighborhood $W$ defined above on which id is locally analytic. The argument used in the proof of Theorem 3.3 of [4] shows that $\tau$ is an analytic isomorphism.

We next introduce the following function diagram:

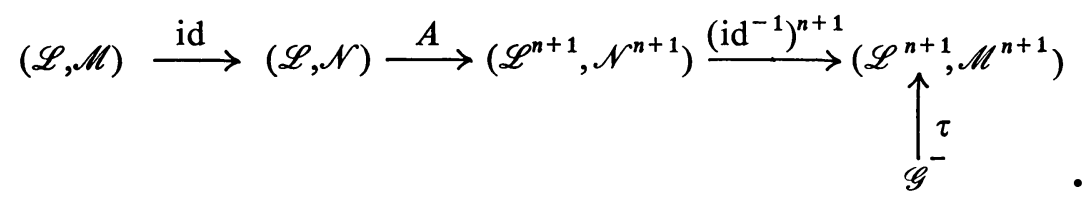

In this diagram, $A$ is defined by $A(x)=\left(p_{0} x, \cdots, p_{n} x\right)$. Since $L_{p_{i}}$ is analytic on $(\mathscr{L}, \mathscr{N})$ for each $i$, it follows that $A$ is analytic. Hence the composite mapping $v \rightarrow \tau^{-1}\left(\mathrm{id}^{-1}\right)^{n+1} A \operatorname{id}(v)$ is locally analytic in $V(v \in V)$. But $\tau^{-1}\left(\operatorname{id}^{-1}\right)^{n+1} A \operatorname{id}(v)=R_{v}$. Hence $C_{R}$ is locally analytic in $V$.

Next we show that $Q$ is globally analytic. Let $x \in \mathscr{L}$, let $K$ be a neighborhood of $x$, and let $b \in \mathscr{L}$, so that $K b \subset V$. Then the mapping

$$
x \stackrel{R_{b}}{\rightarrow} x b \stackrel{C_{R}}{\rightarrow} R_{x b} \stackrel{I}{\rightarrow} R_{x b}^{-1} \rightarrow R_{x b}^{-1} R_{b} \rightarrow R_{x b}^{-1} R_{b}(1)=x^{-1}
$$

is analytic in $K$, since it is the composition of locally analytic mappings. Hence $Q$ is locally analytic everywhere in $\mathscr{L}$, that is, globally analytic. Since $Q=Q^{-1}$, we have that $Q$ is an analytic isomorphism. 
It then follows that id is an analytic isomorphism on $(\mathscr{L}, \mathscr{M})$, since id $=M Q$. Finally $C_{R}$ and $C_{L}$ are globally analytic (again making use of the above diagram); thus the hypotheses of Lemma 5 are satisfied, and the proof is finished.

We conclude with a few remarks motivated by the possibility of generalizing our main theorem. First, the assumption of connectedness may obviously be dropped. Next, we notice, in looking over the crucial steps in the proof, that it was important to prove that id: $(\mathscr{L}, \mathscr{M}) \rightarrow(\mathscr{L}, \mathscr{N})$ was an analytic isomorphism. Therefore, if we wish to give a proof of the theorem without the hypothesis of diassociativity, then we must rely upon means other than diassociativity in order to prove that id is analytic. Lemmas 1-4 all assume diassociativity and were each presented for the sole purpose of proving id analytic. On the other hand, in other parts of the proof of our theorem, diassociativity was not essential; that is, we may state the following corollary to the proof of the theorem:

COROLlary. Let $\mathscr{L}$ be a locally compact, locally connected, connected, finitedimensional loop with a right invariant uniformity and a left invariant uniformity. Let $\mathscr{M}$ be the quotient analytic structure on $\mathscr{L}$ obtained from the group $\mathscr{G}$ - of right translates of $: \mathscr{L}$, and let $\mathscr{N}$ be the corresponding quotient analytic structure on $\mathscr{L}$ obtained from the group $\mathscr{H}$ - of left translates. If id: $(\mathscr{L}, \mathscr{M}) \rightarrow(\mathscr{L}, \mathscr{N})$ is an analytic isomorphism, then $\mathscr{L}$ is a Lie loop.

Indication of proof. One uses Lemma 5 again, as well as the function diagram in the proof of the theorem, and the paragraph preceding this function diagram.

As we have remarked before [4], an example due to Karl H. Hofmann shows that our assumptions of various invariant uniformities cannot be dropped without losing generality. This loop, which can be found on p. 152 of [7], is a commutative, diassociative loop on euclidean 3-space but is not a Lie loop. The Cayley numbers on euclidean 8-space, or the 7-sphere of the Cayley numbers of norm one, provide nontrivial (that is, nongroup) examples where the hypotheses of our theorem are satisfied.

\section{REFERENCES}

1. C. Chevalley, Theory of Lie groups, Princeton Univ. Press, Princeton, N. J., 1946.

2. A. Gleason, Groups without small subgroups, Ann. of Math. (2) 56 (1952), 193-212.

3. S. Helgason, Differential geometry and symmetric spaces, Academic Press, New York, 1962.

4. S. Hudson, Lie loops with invariant uniformities, Trans. Amer. Math. Soc. 115 (1965), 417-432.

5. D. Montgomery and L. Zippin, Topological transformation groups, Interscience, New York, 1955.

6. S. Myers and N. Steenrod, The group of isometries of a Riemannian manifold, Ann. of Math. 40 (1939), 400-416.

7. K. H. Hofmann, Topologische Loops, Math. Z. 70 (1958), 13-37; Topologische Loops mit schwachen Assoziativitätsforderungen, ibid. 70 (1958), 125-155; Topologische Doppelloops, ibid. 70 (1958), 213-230.

SyRaCUSE UNIVERSITY, Syracuse, New York 